\title{
Use of Social Media in Medicine: A Double-edged sword?
}

\section{Fatimah Lateef}

Senior Consultant/ Director of Training and Education, Dept of Emergency Medicine, Singapore General Hospital, Associate Professor Dukes-NUS Graduate Medical School and Yong Loo Lin School of Medicine, National University of Singapore

\section{ARTICLE INFO}

Received : :26/02/2013

Accepted : : : : $: 01 / 09 / 2013$

Published : :01/09/2013

\section{KEYWORD}

Social media,

Medicine,

Communications,

Customer service,

Patient-centric,

Technology,

Internet-based school

\section{ABSTRACT}

Social Media (SM) is changing the way physicians and patients interact. $\mathrm{SM}$ is one of the drivers towards a more patient-centric model of healthcare. It offers a platform for health communications, empowerment and even possibly strengthening the physician-patient relationship. The use of SM in medicine has often brought on a lot of feedback, more negative than positive ones. The academic literature is commonly seen with articles on SM and how it poses dangers and risks to medical personnel. Many SM usage guidelines are also geared towards the expectations of misuse rather than positive considerations and constructive use. There is a need to assess its impact and utilisation in medicine, through processes such as consultation, education and creation of awareness, communications, interaction and sharing of information. The culture of medicine is one that values confidentiality, privacy, one to one interaction and very professional conduct. This is often thought to be contrasting to that of SM, which involves open sharing, connectivity and is thus, more informal. Despite the contrasting cultures, a significant proportion of medical personnel do use or are on SM platforms. Participation in SM networking and other similar internet sites can support physicians' personal expression, enable individual physicians to have presence online, foster collegiality and camaraderie within the profession and provide opportunities to disseminate public health messages and other health communications. If properly and adequately used, within acceptable framework, SM can indeed be a boon, rather than a bane to medicine.

(C) Medical Education Department, School of Medical Sciences, Universiti Sains Malaysia. All rights reserved.

CORRESPONDING AUTHOR: Assoc Prof Fatimah Lateef, Dept of Emergency Medicine Singapore General Hospital Outram Road 1 Hospital Drive, Singapore 169608, Tel: 65 63214972/ 3558, Fax: 6563214873 Email: fatimah.abd.lateef@sgh.com.sg

\section{Introduction}

There are very rapid changes in the communications landscape today. Social media (SM) represents a platform for social interaction and networking, using highly accessible communications techniques. It utilizes webbased and mobile technologies to enable real time interactive dialogues. Social media includes text, audio, video, images, podcasts and other forms of multimedia communications. It has facilitated the speed and breadth of information dissemination, 24 hours a day, growing and evolving rapidly. It is becoming integrated into our businesses, professional and personal lives [1-5]. 
SM is changing the way physicians and patients interact. Patients are now beginning to play a more active role in their treatment. SM is one of the drivers towards a more patient-centric model of healthcare. It offers a platform for health communications, empowerment and even possibly strengthening the physician-patient relationship $[2,4,6]$.

Communications can take place easily, anywhere via a variety of devices these days. Mobile web access via smartphones has overtaken conventional desktop internet use. Smartphones can be used to access emails and social networking sites on the go. In coffee-shops and subway stations people are often in virtually synchronous conversations via instant messaging. No spoken words are used strictly and it may seem that the wealth of emotions will be lacking. However, shorthand expressions, pictograms and emoticons are utilized to express feelings via these electronic communications modes.

Medicine, as practiced by physicians, is the art of caring and healing, which utilizes applied sciences to arrive at diagnoses, treatment and preventive aspects of illnesses. The use of SM in medicine has often brought on a lot of feedback, mostly negative $[1-3,6]$. The academic literature is commonly seen with articles on SM and how it poses dangers and risks to medical personnel [26]. Many SM usage guidelines are also geared towards the expectations of misuse rather than positive considerations [5-8]. There is really a need to assess its impact and utilisation in medicine, through processes such as consultation, education and creation of awareness, communications, interaction and sharing of information. The culture of medicine is one that values confidentiality, privacy, one to one interaction and very professional conduct. This is often thought to be contrasting to that of SM, which involves open sharing, connectivity and is thus, more informal. Despite the contrasting cultures, a significant proportion of medical personnel do use or are on SM platforms. Participation in SM networking and other similar internet sites can support physicians' personal expression, enable individual physicians to have presence online, foster collegiality and camaraderie within the profession and provide opportunities to disseminate public health messages and other health communications. If properly and adequately used, within acceptable framework, SM can be harnessed in positive ways to bring forth advantages to the practice of medicine today $[1,3,4,9]$.

\section{Responsible Use of Social Media}

As the practice of medicine involves dealing with very private and intimate information of people, it is crucial to ensure maintenance of confidentiality at all times. Factual or education comments made by physicians using SM must be accurate as the public will tend to take these as the truth, especially when they are made by professionals. During interaction, colleagues and friends must be treated with respect and there should not be belittling or 'looking down' upon other medical professionals. When using SM, physicians must be aware of the potential impact and the vastness of its outreach. They must also be aware that the contents can be shared rapidly, on a variety of platforms. Users of SM are not passive in reading information. They have the ability to directly influence generation of information and can act as local hubs within their own communities, to propagate messages and ideas. With such breadth of exposure, conflict of interest can arise as well [2, 3, 9-11].

Physicians using SM should review their privacy settings regularly to ensure its appropriateness. They must be able to draw the line between their personal and professional involvement. If a physician is going onto SM platforms to educate the public, they can certainly do so in their professional capacity. They should be forthcoming with their credentials, certification and employment. However, many do tend to avoid requests for personalized medical advice on these platforms $[2,3,9,12]$. The public accessing SM sites are often able to view interactions, postings and message by physicians and will form their own ideas and perceptions of the profession. Some do also source for specialists or doctors through such sites. Thus, it 
is even more crucial for physicians to share accurate and appropriate information.

In recent years the healthcare fraternity has increasing awareness of the opportunities and challenges posed by social media. Medical boards in institutions usually issue guidelines on SM use for physicians and staff. The board will also have the authority to discipline staff in the event of unprofessional behavior and the inappropriate use of SM. Disciplinary action can range from a letter of reprimand to the revocation of practice license in serious cases [5, 7-9, 12]. Indeed, ethical and professional standards must be applicable at all times and in all aspects of physicians' practice.

$\mathrm{SM}$ is much too prominent for physicians to ignore. It is here to stay and will continue to evolve. SM does have its positive aspects, such as facilitating relationships between colleagues and providing a framework for expression. However, physicians will need to be careful not to inadvertently put forth personal information which may cross the boundary into advertising. Going into dual relationships with patients should also be avoided. Even when putting information across, without revealing the exact names and details of patients, caution is required because at times the circumstances and information (e.g., type of condition, injuries, location in an institution etc) potentially may allow someone in the community to identify the patient, thus losing confidentiality [13-16].

Using SM and electronic communications has a lot to do with the process and attitude of the communicator. The exact meaning of comments can be misconstrued when people use SM inappropriately without giving much thought to the consequences of their comments. Users must be mindful of the needs and feelings of others. There is a need to think through what one is posting and how it might be interpreted.

\section{Benefits of SM in the Practice of Medicine}

SM in medicine is a valuable intelligent tool, a customer service tool and has the potential to lead to better patient care and outcomes $[16,17]$.

\section{Intelligent Tool}

Engagement and surveillance on SM enables physicians to understand what patients are concerned about and going through to improve their health. What are their obstacles to improving their health and what health issues interest and concern them. Scanning SM also gives physicians a rough idea of the healthcare needs of the community. Certain health awareness trends and practices can also be discovered. When the public do discuss their illness states online as well, certain 'hot spot' during early phases of outbreaks, can be identified or suspected early. SM has the potential to empower patients to get more information, ask questions and receive answers to their health related concerns. This is in alignment with patient-centric care.

Smartphones have significant potential to make faster and better contact with Emergency Medical Services (EMS). This also includes assisting caller with step by step instructions for cardiopulmonary resuscitation (CPR). The technology also allows users to locate the position of the nearest automated external defibrillator (AED). OpenGeoSMS and iPhone's Global Positioning Systems enable communications on location coordinates and content between 'location-aware' devices, using short message service (SMS) [17].

Google People Finder is yet another example of the power of the internet and social media to connect people after disasters. The website started by Google engineers is now very much a part of the Google Crises Response Division of Google.org. People can access the site via mobile devices or traditional internet [18]. The International Network of Crisis Mappers is also another example where experts, practitioners, researchers and volunteers engage in discussions on humanitarian crises, crisis technology and crisis mapping. These people leverage on mobile and web-based technology to assist communities prone to disasters in a variety of ways [19]. 


\section{Customer Service Tool}

When certain inaccurate perceptions or comments are made on SM, there is the potential to correct these immediately, within a short time span. Addressing complaints on SM is also faster and may allow a dialogue to go on to correct misperceptions or misunderstanding. However, there is a need for caution, such that specific details and sensitive medical information are kept offline. The online discussion allows others to see the expected course of action and proactive move institutions or clinics are taking The public will also understand how seriously these institutions manage their customer service issues. Other uses include targeted communications with patients, response to queries and also clarifications. Institutions are now utilizing SM to interact with their patients and clients on a wide variety of issues [20,21].

\section{Quality of Care and Outcomes}

With SM, institutions can now compare how they are doing and what services they provide, with other institutions. This can be at the national, regional or international levels. Certain standards which are publicized can also be looked at and when institutions are planning certain facilities or strategies they can learn from the experience of others who have already embarked on these. Responses to quality related issues raised online allow a more rapid response. Time for institutions to respond to complaints and queries can be a good assessor of service quality standards [20, 21].

With the advent of technology, moving out of the traditional practices can be facilitated by use of SM. For example blood sugar monitoring for diabetic patients, electrocardiogram monitoring for patients with ischemic heart diseases, peak flow monitoring in asthmatic and chronic obstructive lung disease patients can now be monitored by physicians and staff online or electronically. This can save patients having to make multiple trips to the clinics or hospitals. This practice can potentially improve compliance as well as it is easier and more convenient for patients. They also avoid long waits with this strategy. Patients can also be reminded of their appointments online and through SM instead of having letters send traditionally or having to employ specific staff to make reminder phone calls $[11,17,21]$.

IT and SM continues to play a prominent role in improving patient care. The information shared must allow them to make informed choices and decision related to their health. At Singapore General Hospital and Singhealth Cluster in Singapore, the use of online appointment systems is noted to show a lower default rate amongst patients. Other award winning applications include "Singhealth Health Buddy" which offers health tips and e-library services on medical conditions and their treatment. Mobile@SGH is another application which offers easy access to payments details and cost as well as the Emergency Department queue viewer. At the Kandang Kerbau Women and Children's Hospital, the Women and Child Health Pedia applications which is very popular, comes with a pregnancy calendar and calculator as well as a planner to track fetal development

This century, we are also managing the evolution of the e-patient, who can act online as advocates, helping others to join in discussions and be more empowered. SM has now been observed to be utilized through various stages of diseases for example, for diagnoses and information gathering, sourcing for provider and getting second opinion, acceptance of diagnoses and joining online support groups, participation in various types of research and trials.

For physicians, SM provides a channel to share experiences in closed peer-to-peer networks, discussing the latest research and findings, trials and even best practices. The online social network is able to multiply these peer networks many times over and even across borders internationally. The expanded network can also highlight differences in practice across institutions or geography. 


\section{Social Media, Continuing Medical Education (CME) and Lifelong Learning}

The amount of medical information required for medical practice these days have increased exponentially. New evidence-based findings, bench to bedside research and innovations are all increasing at a rapid rate. For a physician to keep abreast, it is almost inevitable, he will realize the limitations of just using traditional techniques of information and knowledge acquisition. Physicians and healthcare personnel will have to adapt to a new model of learning and sharing information.

Many institutions these days are using SM to conduct CME. It has proven to be easily accessible, popular and time-saving for the busy physician and is catching on. In this sense as well, SM can help to improve the quality and standard of patient care [22-24].

Institutions and healthcare staff associations should provide guidance on the use of SM. Proper strategies and explanation can increase understanding of what the practice is all about. The more youthful users are already technology savvy and the older ones may require some degree of assistance and instructions [22-25].

Currently, there is a lack in the number of studies with regards to physicians' attitude, interest and concerns on the use of SM in healthcare. These studies will be able to shed more light on their understanding, preferences, barriers as well as concerns $[24,25]$.

\section{Research and Publications}

Use of SM in medical research is now moving from experimental pilots to informed and strategic participation. Many companies and institutions are exploring new means to use SM to support various aspects of clinical trials e.g. recruitment, follow up. Some current tactics in use include SM advertising such as on Facebook and search engine marketing. These can be useful in international trials and helps with faster trial recruitment, especially in studies of very rare diseases.
Communication barriers and outdated technology can hinder applying evidence-based research findings in a timely manner. SM facilitates sharing of information and findings in this aspect. For example, Williams, in his paper stated that "the facebook fanpage accounted for the largest number of referrals and consults to the Science of Soccer Online (SSO) website. Those assessing the page spend an average of 5 minutes and read two articles per visit". New findings and evidence which are practical are easily shared and read. This outreach to practitioners is easy, rapid and hassle free [2628].

SM also allows the creation of a virtual community of academics, practitioners and even interested patient groups to have ongoing conversations and discussions. These platforms can be used to share best practices, information, publicize events, seminars and conferences. Others have also tried recruitment of candidates for participation in trials. It is important to do this in an appropriate, ethical way as one of the commonest problems is that the researchers utilize their own accounts to collect data. The safest way would be to use an account that has "no friends" or a specially set up account for this purpose. In recruiting using this technique, some may not be aware that SM users are not necessarily representative of any larger groups $[26,27]$.

SM has also been used for discussions on sports, injuries and treatment, different types of cancers, traditional medicine practices and even chronic illnesses such as rheumatoid arthritis and systemic lupus erythematosus. SM provides a sharing platform in these circumstances. There are also discussions by thought leaders on certain topics but these can at times be amongst a closed circle of people only. Networking and advocacy too is facilitated by use of SM. In publications, SM allows journal screening, choice and review of impact factors. These days more and more journals have electronic or online versions. This makes journals even more accessible and promote dissemination of information and research findings. More journals are also going the 'open access' way and report increase in 
readership traffic and number of "hits". This also facilitates the reviewing process. Whereas it used to take months for reviews to be completed, now it may take between days to weeks only with electronic communications and use of new media [26-29].

\section{Conclusion}

The Successful SM taps on the power of mass collaboration through user participation. However with the degree of transparency it provides, users are privy to each other's involvement. They will get to use, reuse, recycle, augment critique and even rate one another's contributions. SM in its own way propels its own advancements, it is important to balance use of SM with the more traditional forms of communications. Face to face communications is still very highly relevant in Medicine.

SM is not just for IT-trained persons. It's for everyone. In healthcare, its existence is already making transformation. The future will see more health-related applications, blogs and web-based technology amongst a more educated patient base, who are more empowered and more knowledgeable. The SM revolution is here and is happening for real. It's challenging but refreshing and will certainly change the way physicians of this century think, act and practice Medicine.

\section{Reference}

1. Van De Belt TH, Eyelen LJ, Berben SA et al. Definition of Health 2.0 and Medicine2.0: A systematic review. J Med Internet Res 2010; 12(2): e18

2. Timimi FK. Medicine, morality and health care social media. BMC Med 2012; 10: 83

3. Decamp M. Physicians, social media and conflict of interest. J Gen Intern Med 2012; Epub

4. Greysen SR, Kind T, Chretien KC. Online professionalism and the mirror of social media. J Gen Intern Med 2010; 25(11): 1227-9

5. GMC Draft Guidelines. Available at https://gmc.e-
consultation.net/econsult/consultation_Dtl.asp $\mathrm{x}$ ?consult_Id-271\&status

6. George DR, Green MJ. Beyond good and evil: explaining medical trainees use of social media. Teaching and Learning in Med 2012; 24(2): 155-7

7. Shore R, Halsey J, Shah K et al. Report of the AMA Council on ethical and judicial affairs: professionalism in the use of social media. The Journal of Clin Ethics 2011; 22: $165-72$

8. Gosbell A, Killen A, McCarthy S. Australasian College for Emergency Medicine. Emergency Medicine Australasia and social media. Emerg Med Australia 2012; 24(40: 463-4

9. Bosslet $\mathrm{G}$, Torke A, Hickman $\mathrm{S}$ et al. The patient-doctor relationship and online social media networks: Results of a national survey. J Gen Int med 2011; 26: 1-7

10. Moubarak G, Guiot AI, Benhamou Y et al. Facebook activities of residents and fellows : impact on the patient-doctor relationship. J Med Ethics 2011; 37: 101-4

11. Jain SH. Practising medicine in the age of Facebook. NEJM 2009; 361: 649-51

12. Lagu T, Kaufman E, Asch D et al. Contents of web-blogs written by health professionals. Journal of Gen Int Med 2008; 23; 1642-6

13. Victorian B. Nephrologist using social media connect with far-flung colleagues, healthcare consumers. Nephrology Times 2010; 3: 1-18

14. Chretien KC, Goldman EF, Beckman L et al. Its your own risk: medical students' perspective on online professionalism. Academic Med 2010; 85: S68-S71

15. Guseh J, Brendel R, Brendel D. M edical professionalism in the age of online social networking. Journal of Medical Ethics 2009; 35: $584-6$

16. Landman M, Shelton J, Kauffmann R et al. Guidelines for maintaining a professional compass in the era of social networking. $\mathrm{J}$ of Surg Education 2010; 67: 381-6

17. Rimal RN. Tapping into the social network. Bull World Health Organ 2012; 90(6): 410-1

18. Google Person Finder @ http://www.google.org/personfinder/global/h owitworks 
19. Crisis mappers @ http:crisismappers.net/

20. Bendall- Lyon D, Paners TL. The role of complaint management in the service recovery process. The Joint Commission on Accreditation of healthcare organisations, 2001; 27(5): 278-86

21. McGowan BS, Wasko M, Vartabedian BS et al. Understanding the factors that influence the adoption and meaningful use of SM by physicians to share medical information. J Med Internet Res 2012; 14(5): e117

22. Davis DA, Thomson MA, Oxman AD et al. Changing physician performance: a systematic review of the effect of continuing educational strategies. JAMA 1995; 274 (9):700-5

23. Azu MC, Lilley EJ, Kolli AH. Social media, surgeons and the internet: an era in an error?. Am Surg 2012; 38(5): 555-8

24. Wang AT, Sandhu NP, Wiltich CM et al. Using social media to improve continuing medical education: a survey of course participants. Mayo Clin 2012; 87(12): 116270

25. George DR, Dellasega C. Use of SM in graduate level medical humanities education: Pilot studies from Penn State Collage of Medicine. Med Teach 2011; 33(8): e429-34

26. Nair V, Khan $S$, Jhaveri KD. Interactive journals and the future of medical publications. AM J Med 2012; 125(10): 1038-42

27. Williams JH. Use of SM to communicate Sports Science Research. Int J Sports, Science and Coaching 2011; 6(2): 295-300

28. Farmer AD, Bruckner Holt CE, Cook MJ et al. Social networking sites: a novel portal for communications. Postgrad Med J2009; 85:455-9

29. Correspondence: The power of social networking in Medicine. Nature Biotechnology 2009; 27(10): 888-90 